\title{
Occurrence of lactation problems versus socio-demographic conditions. Initial findings
}

\author{
Grażyna Bączek', Nela Kameduła', Wojciech Giermaziak² \\ 'Department of Gynaecology and Obstetrics, Medical University of Warsaw, Warsaw, Poland \\ ${ }^{2}$ The Stanisław Konopka Main Medical Library, Warsaw, Poland
}

\begin{abstract}
Introduction: Breastfeeding is the best method of feeding newborns and babies. The percentage of Polish women who feed their babies only with breast milk at the age of six months is unsatisfactory. The impact of social and demographic factors on breastfeeding-related behaviour was described, so an attempt to identify specific risk groups in relation to age, marital status, education level, or place of residence seems justified.

Material and methods: A diagnostic survey covered a group of 256 women who gave birth to a baby in the last 6 months by a Caesarean section in a hospital in Warsaw, however not earlier than in the $37^{\text {th }}$ week of pregnancy.

Results: Women living in towns/cities with a population of over 50,000 people more often supplementarily fed their babies with modified milk. Mothers aged 18-25 years mentioned lactation problems far less often than mothers representing other age groups. Women of that age most often denied having problems with breast milk scarcity. The problem was most common in mothers over 35 years of age. No statistically significant differences in the frequency of reporting problems related to sore nipples were observed. The ailment was most often reported by women aged $18-25$ years.

Conclusions: Socio-demographic conditions are related to the behaviour that affects breastfeeding. The particularly important factors include the mother's age and education. Every group experiences different problems caused by various factors. Mothers representing the group with the lowest education level should be provided with special care.

Key words: breastfeeding, lactation, socio-demographic factors, lactation disorders.
\end{abstract}




\section{Introduction}

Breastfeeding is the best method of feeding newborns and babies. It brings a number of health benefits for the mother and baby [1]. Global authorities unanimously claim that this is the feeding method that should be strived for. The American Academy of Paediatrics recommends feeding babies only with breast milk for the first 6 months of their lives [2]. Numerous initiatives to promote breastfeeding have brought the expected result. Poles are aware of the advantages of natural feeding and are eager to follow the recommendation. Ninety-one percent of mothers-to-be declare such a will. According to data from 2014, $98 \%$ of babies are breastfed in the first period of their lives [3]. Nevertheless, the percentage of Polish women who feed their babies only with breast milk in the sixth month of their age is highly unsatisfactory [2]. After 6 weeks it drops to $46 \%$ to reach only $42 \%$ between the second and sixth month of age [3]. Women who had Caesarean section represent the group in which the risk of giving up breastfeeding prematurely is the highest [4]. In relation to the growing percentage of Caesarean sections, the group has been growing every year [5].

The paper describes the impact of socio-demographic factors on breastfeeding behaviour [6-9]. An attempt to identify specific risk groups in relation to age, marital status, education level, or place of residence is then justified. Knowledge of the issue can help to develop detailed programmes (on a regional level in particular) intended to support breastfeeding. The purpose of the study is to try to identify risk groups based on the frequency of occurrence of lactation problems among women divided based on socio-demographic criteria.

\section{Material and methods}

The study was carried out in 2017 using a diagnostic survey method. A proprietary questionnaire containing questions on the course of lactation a few days after giving birth was the study tool. Women who gave birth in the last

A

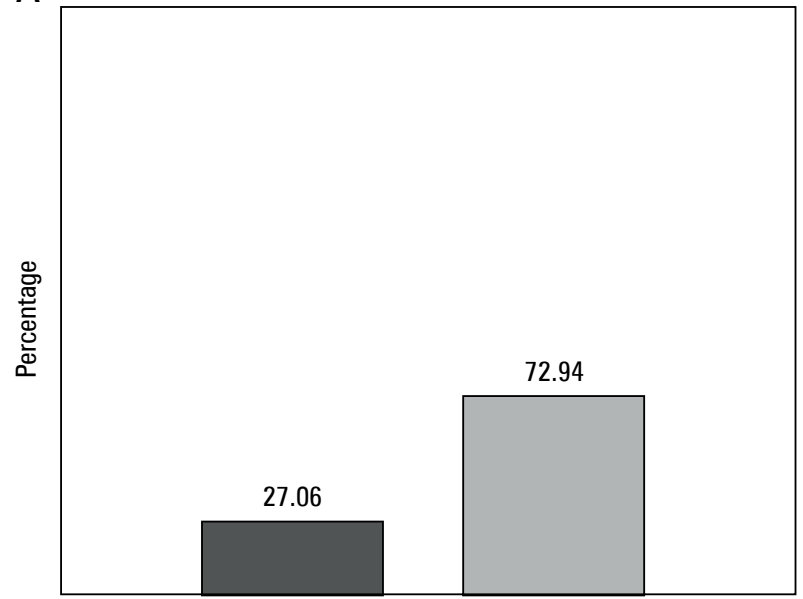

No

Yes
6 months by means of Caesarean section in a hospital in Warsaw, but not earlier than in the $37^{\text {th }}$ week of pregnancy, were invited to participate in the study. The participation was voluntary, and all respondents remained anonymous. The study group consisted of 295 women. Following an initial analysis, 37 were excluded from the study. The exclusion criteria included incorrectly filled questionnaires, delivery in a hospital outside Warsaw, and delivery before the $37^{\text {th }}$ week of pregnancy. Finally, 257 women were qualified for the study; 93 of them filled out an online questionnaire, while 164 filled out paper questionnaires. Patients staying in obstetrics and neonatal units in hospitals filled out the questionnaire. The hospital management's consent to carry out the study was the criterion for selecting the centres. Electronic questionnaires of the same content were made available for all centres at the same time. The questionnaires were filled out by members of social media groups associating mothers who gave birth by Caesarean section. All respondents expressed their informed consent to participate in the study.

\section{Statistical analysis}

The obtained data were subject to statistical analysis using Microsoft Office Excel. The statistical method utilised a $\chi^{2}$ test. The assumed significance level was $a<0.05, p<a$. The results of the statistical analysis are presented in Table I.

\section{Results}

The study group consisted of 256 women. Women aged 31-35 years constituted the largest part of the group (35.5\%). The proportion of women aged 26-30 years was nearly the same (34.5\%). Women over 35 years old constituted $18.8 \%$. The smallest group was represented by $18-25$-year-old women $(11.3 \%)$. There were no women under 18 years old in the study population.

$77.3 \%$ of the respondents declared having university degrees, while $20.3 \%$ completed secondary education. The smallest groups were represented by women with basic vocational (1.6\%) and primary $(0.8 \%)$ education. Due to a low

B

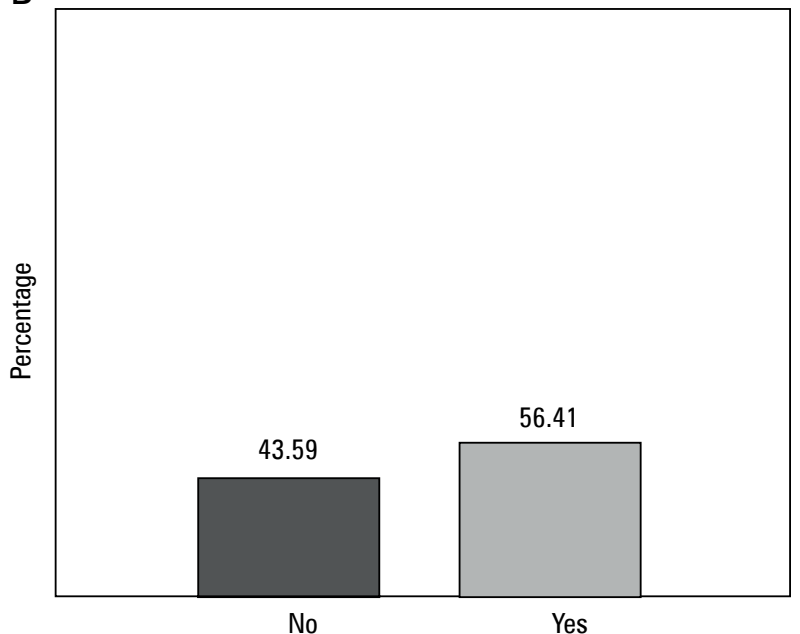

Figure 1. Attitude to the statement My baby was supplementarily fed with modified milk when staying in hospital ( $n=257)$ : A - city with a population $>50,000$ people, $\mathbf{B}-$ town with a population $<50,000$ people or village 
Table I. Statistical significance ( $p 1)$ of differences between variables

\begin{tabular}{|l|c|c|c|c|}
\hline Variables & Mother's age & Education & Place of residence & Marital status \\
\cline { 2 - 5 } & & Number for all variables N = 257 & 0.750625555 \\
\hline $\begin{array}{l}\text { Supplementary feeding } \\
\text { of the baby }\end{array}$ & 0.422605341 & 0.646756538 & 0.037274407 & 0.60277111 \\
\hline Lactation problems & $3.45 \times 10^{-34}$ & $6.03 \times 10^{-175}$ & 0.734861141 & 0.577095 \\
\hline Too little breast milk & 0.027769 & 0.784457 & 0.052905 & 0.390483 \\
\hline Sore nipples & 0.153124 & 0.059049 & 0.480765 & 0.414835 \\
\hline $\begin{array}{l}\text { Difficulty in getting the } \\
\text { baby to latch }\end{array}$ & 0.15545 & 0.918328 & 0.940057 & \\
\hline
\end{tabular}

number of women in the groups with primary and basic vocational education, they were classified as one group.

The majority of the respondents were married women living in towns/cities with a population of over 50,000 (84\%). Sixteen percent of the respondents were inhabitants of villages or towns with a population of less than 50,000. $80.5 \%$ of the respondents were married. Unmarried women

A

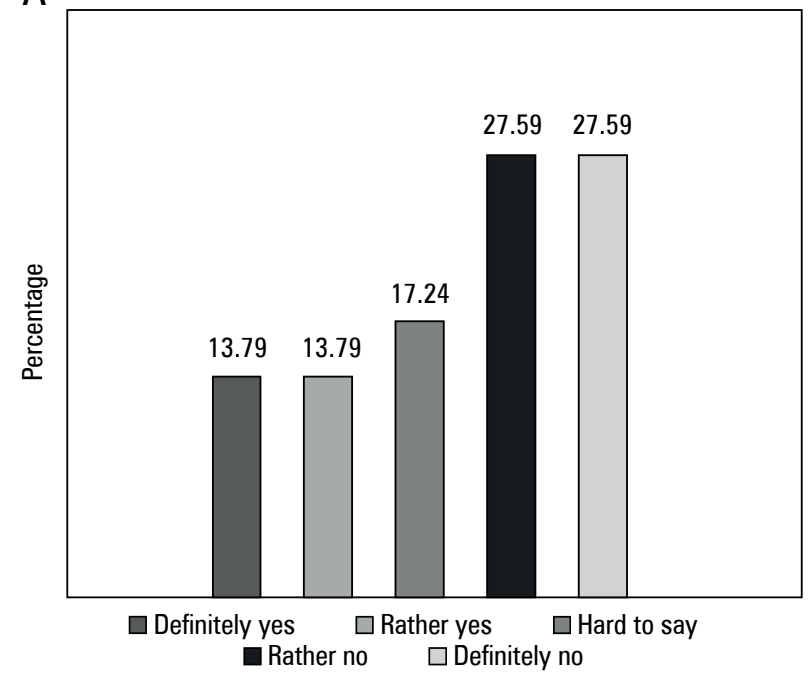

C

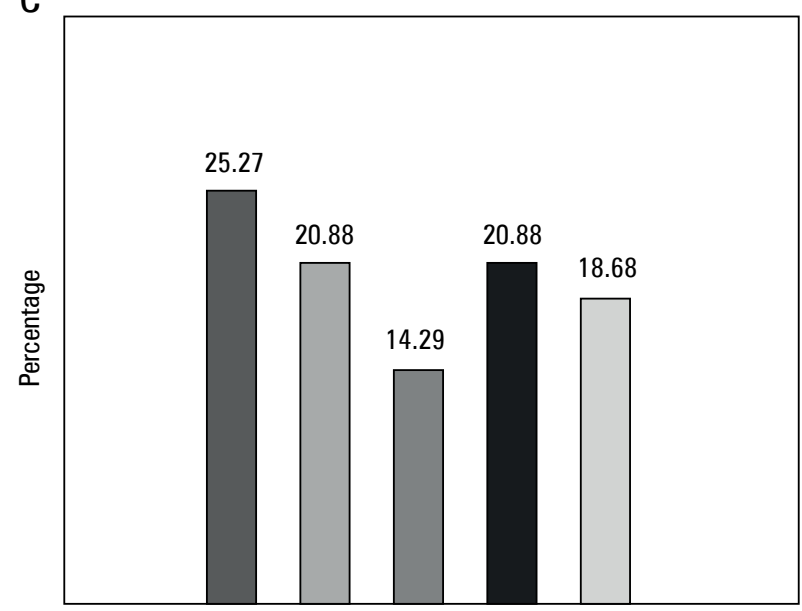

$\square$ Definitely yes $\square$ Rather yes $\square$ Hard to say

- Rather no $\square$ Definitely no constituted $18 \%$, divorced women $1.2 \%$, while the share of widows amounted to $0.4 \%$. A decision was made to stick to two groups divided according to their marital status. One group included unmarried women (18\%), while women with a different marital status formed the other group (82.1\%).

$94.9 \%$ of the respondents agreed that breastfeeding is the best way to feed newborns and babies. $95.3 \%$ wanted to B

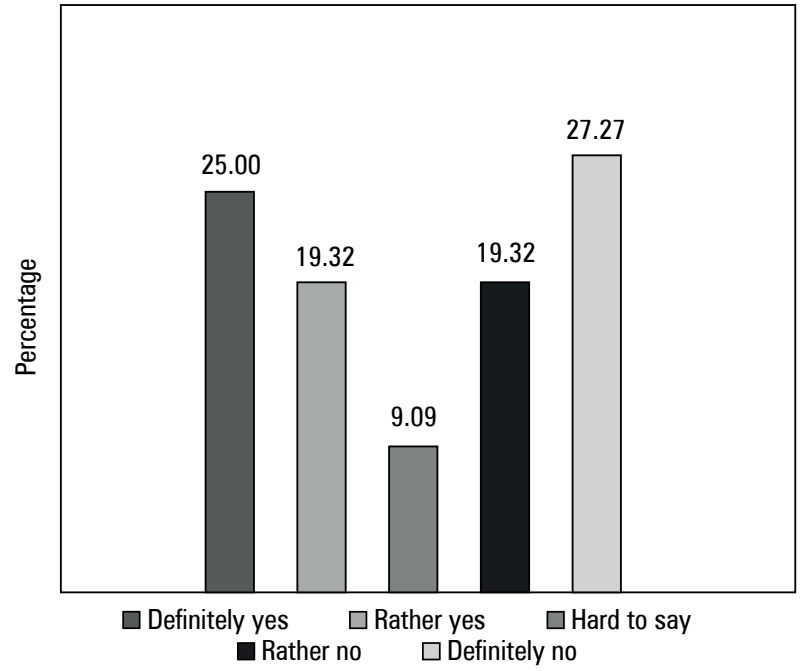

D

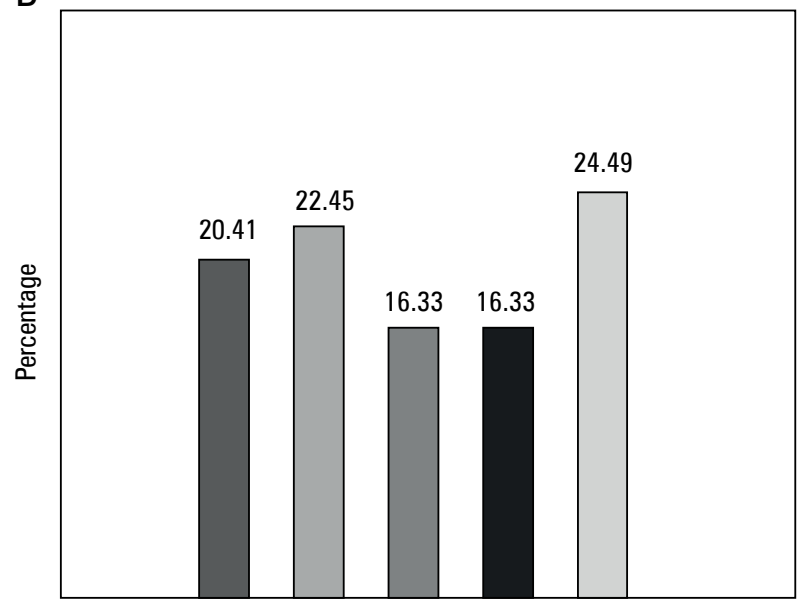

$\square$ Definitely yes $\square$ Rather yes $\quad \square$ Hard to say

$\square$ Rather no $\square$ Definitely no

Figure 2. Attitude to the statement I had lactation problems when staying in hospital. Distribution of answers according to the respondents' age $(n=257)$ : $\mathbf{A}-18-25$ years old, $\mathbf{B}-26-30$ years old, $\mathbf{C}-31-35$ years old, $\mathbf{D}->35$ years old 
breastfeed their babies. The respondents were asked if their babies were supplemented with modified milk when staying in hospital. $70 \%$ gave a positive answer, while $30 \%$ gave a negative answer. Statistically significant differences were observed between the frequency of supplementary feeding of babies among women living in towns/ cities with a population of $>50,000$ and those living in smaller towns/villages ( $p 1=0.037274407, p 1<a)$. Women living in bigger towns/ cities supplemented their babies with modified milk more often (Figure 1). No other statistically significant differences were observed between socio-demographic factors and the frequency of supplementary feeding of babies when staying in hospital (Table I). The respondents were asked to express their opinion on the following statement: I had lactation problems when staying in hospital. The number of women who said Definitely yes (22.5\%) and Definitely not (24\%) was nearly the same. A similar number of respondents said either Rather yes (19.8\%) or Rather not (20.6\%). 13.4\% of the respondents chose the Hard to say answer. Statistically significant differences were observed between the respondents'

Table II. Attitude to the statement I had lactation problems when staying in hospital. Distribution of answers depending on the respondents' age $(N=257)$

\begin{tabular}{|l|c|c|}
\hline Age group [years] & $\begin{array}{c}\text { Total of answers (\%) } \\
\text { Definitely not and Rather not }\end{array}$ & $\begin{array}{c}\text { Total of answers (\%) } \\
\text { Definitely yes and Rather yes }\end{array}$ \\
\hline $18-25$ & 55.18 & 27.58 \\
\hline $26-30$ & 46.59 & 44.32 \\
\hline $31-35$ & 39.56 & 46.15 \\
\hline$>35$ & 40.82 & 42.86 \\
\hline
\end{tabular}

A

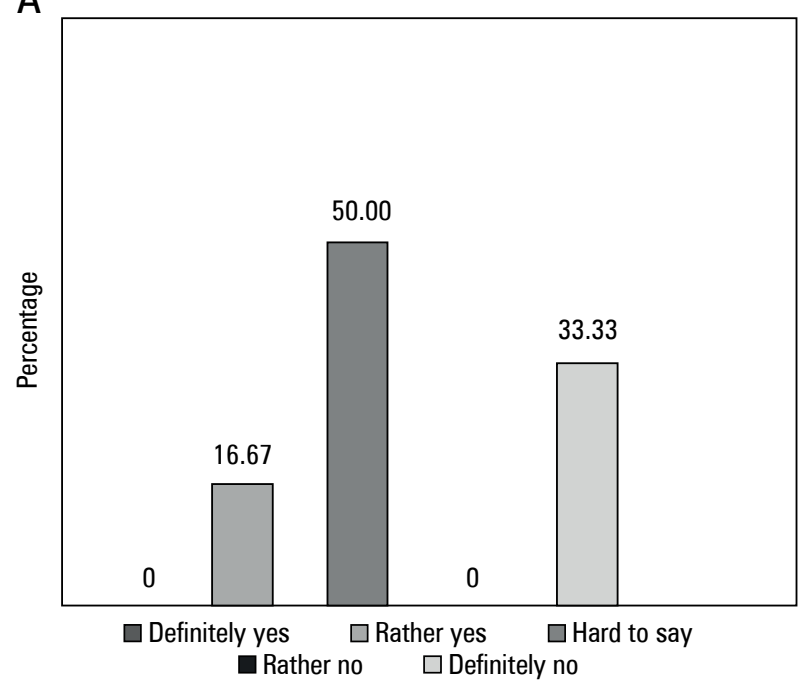

C

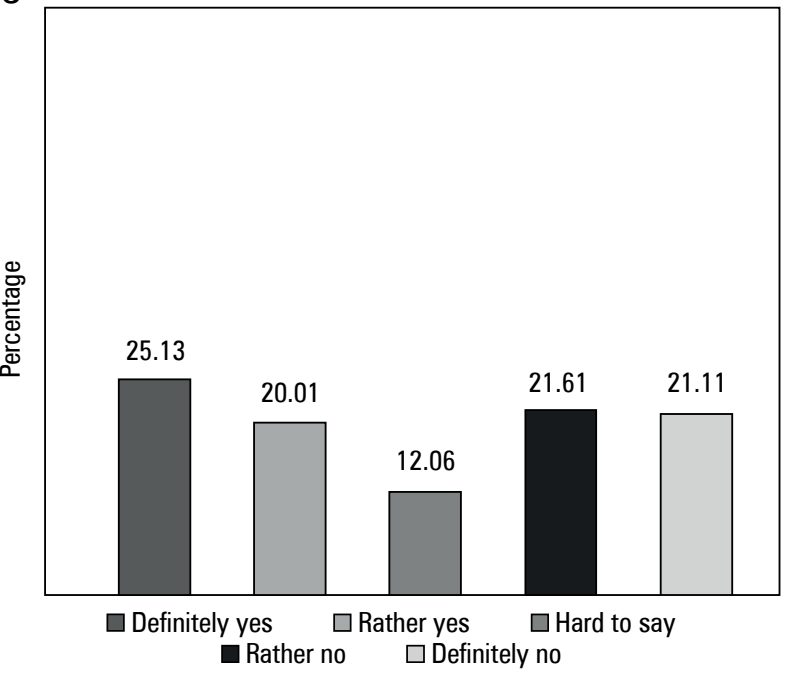

B

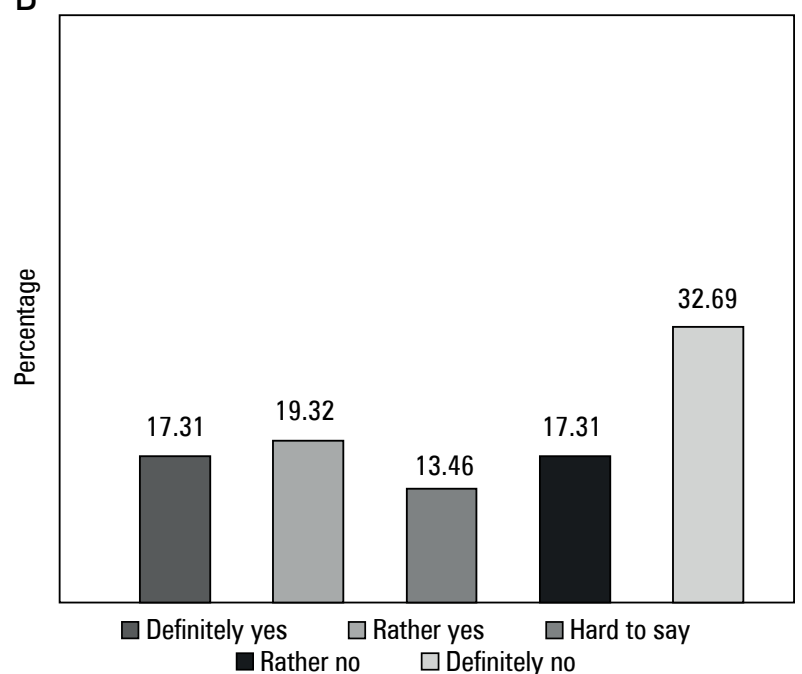

Figure 3. Attitude to the statement I had lactation problems when staying in hospital. Distribution of answers according to the respondents' education $(n=257)$ : A - primary/basic vocational, B - secondary, C - tertiary 
age and the frequency of reporting lactation problems ( $p 1$ $\left.=3.45 \times 10^{-34}, p 1<a\right)$. Lactation problems were most often reported by women aged $31-35$ years. $46.15 \%$ of respondents in this group confirmed having lactation problems (the total of answers Definitely yes and Rather yes). $39.56 \%$ of the respondents denied having any lactation problems (the total of answers Definitely not and Rather not). Similar answers were given by women aged $26-30$ years. $44.32 \%$ said that they had had lactation problems (the total of answers Definitely yes and Rather yes). However, extreme answers were most common in this age group (Definitely yes and Definitely not). The percentage of hesitant persons who answered Hard to say was the lowest in this age group (9.09\%). Lactation problems were least often reported by respondents aged 18 25 years. $55.18 \%$ of them did not have lactation problems. $27.58 \%$ of women in the aforementioned age group experienced lactation problems. The greatest difference in the frequency of giving positive and negative answers was also observed in this age group (Figure 2). Table II presents the observed relationship: in the case of respondents aged 18 to
35 the frequency of lactation problem occurrence is directly proportional to age. Statistically significant differences were also observed for the frequency of lactation problem occurrence depending on education $\left(p 1=6.03 \times 10^{-175}, p 1<\mathrm{a}\right)$. Fifty percent of the respondents who completed primary or basic vocational education were not able to say if they had lactation problems (Hard to say answers). Women in this age group were the ones who chose extreme answers most often (Definitely yes/no). The greatest difference was observed between the percentage of women reporting lactation problems and those denying them. Only $16.67 \%$ of women confirmed having lactation problems. The percentage was much higher in the case of women with tertiary (45.23\%) and secondary (36.54\%) education. The difference in the number of women with tertiary education who gave positive and negative answers was minor (45.23\% and $42.72 \%$, respectively) (Figure 3). The respondents also replied to the statement I had little breast milk. The most common answer was Definitely yes (26.1\%). The second most popular answer was Definitely not

B

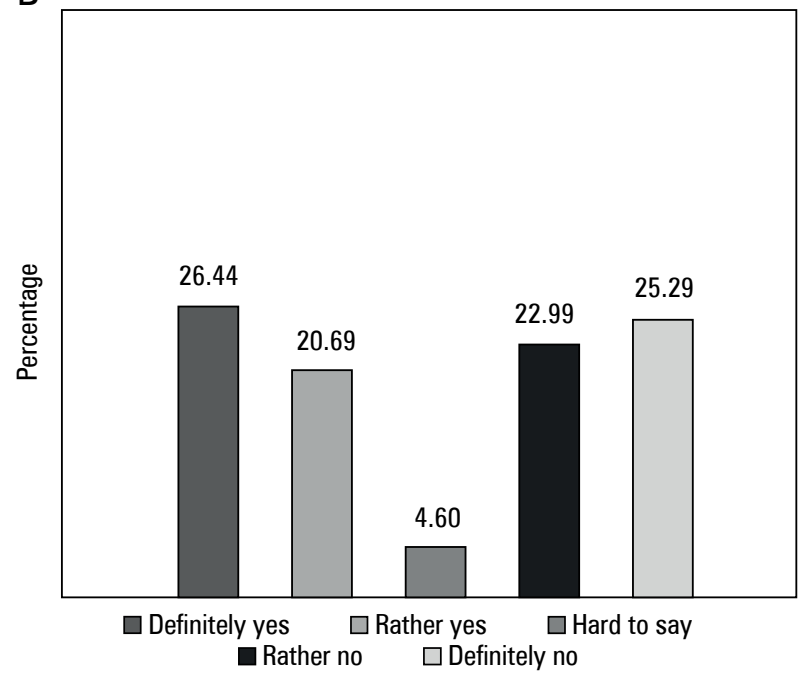

D

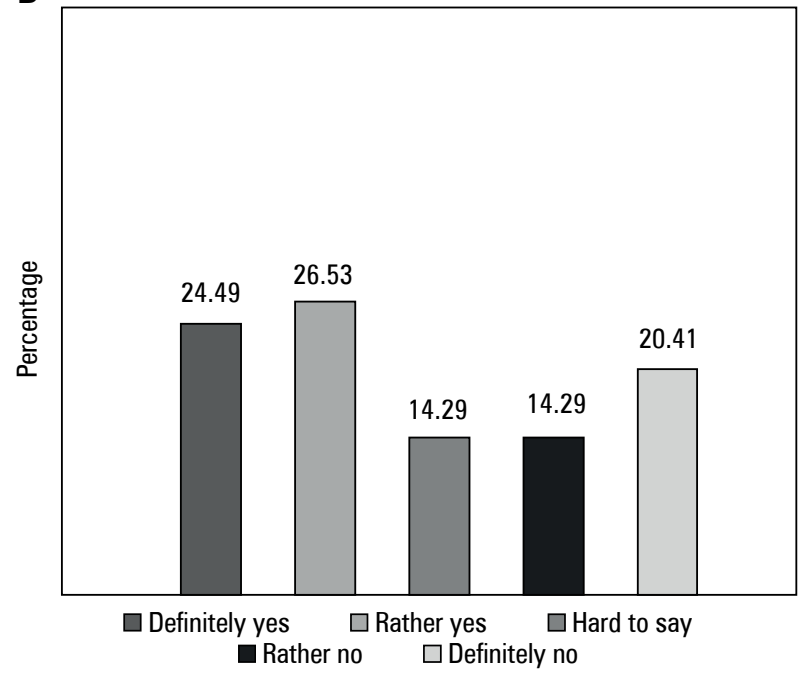

Figure 4. Attitude to the statement $I$ had little breast milk. Distribution of answers depending on the respondents' age $(n=257)$ : A - 18-25 years old, B - 26-30 years old, $\mathbf{C}-31-35$ years old, $\mathbf{D}->35$ years old 
A

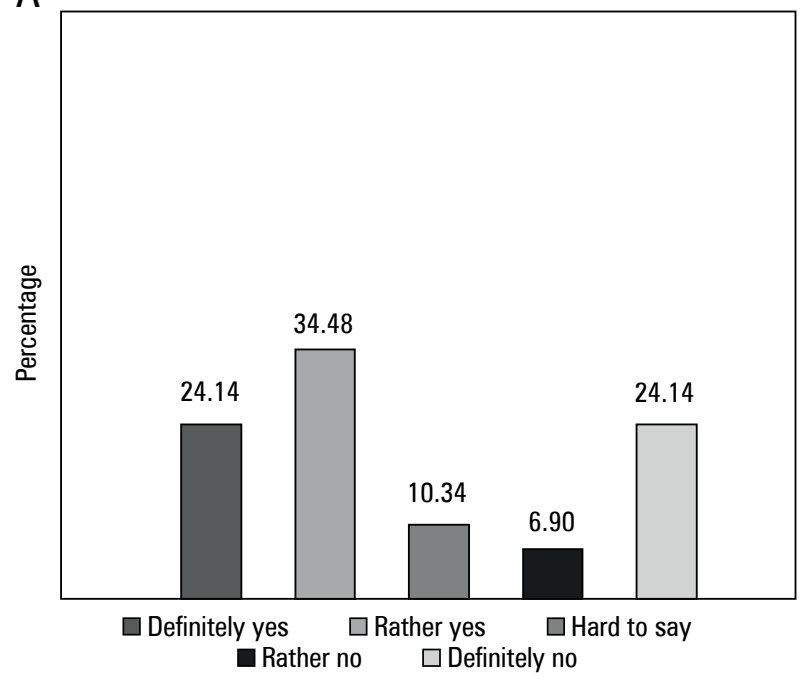

C

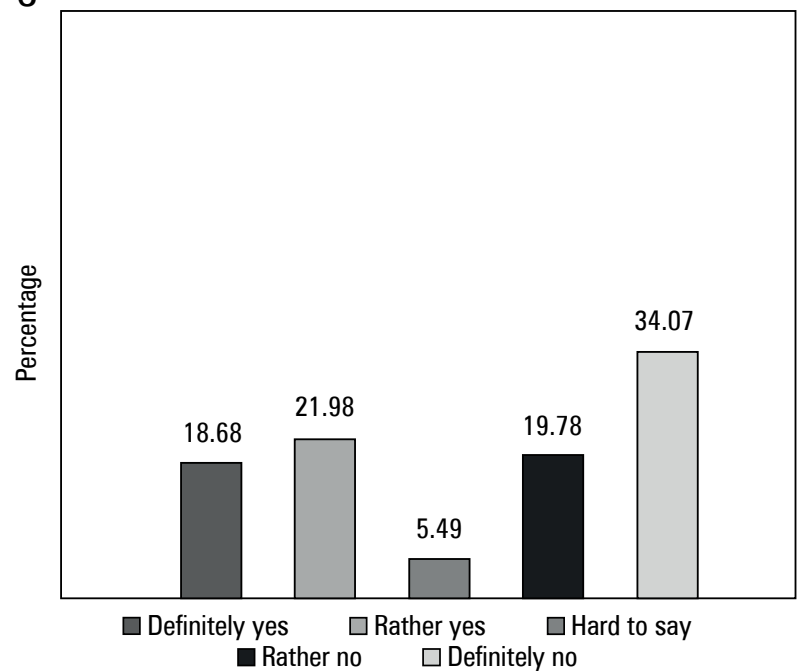

B

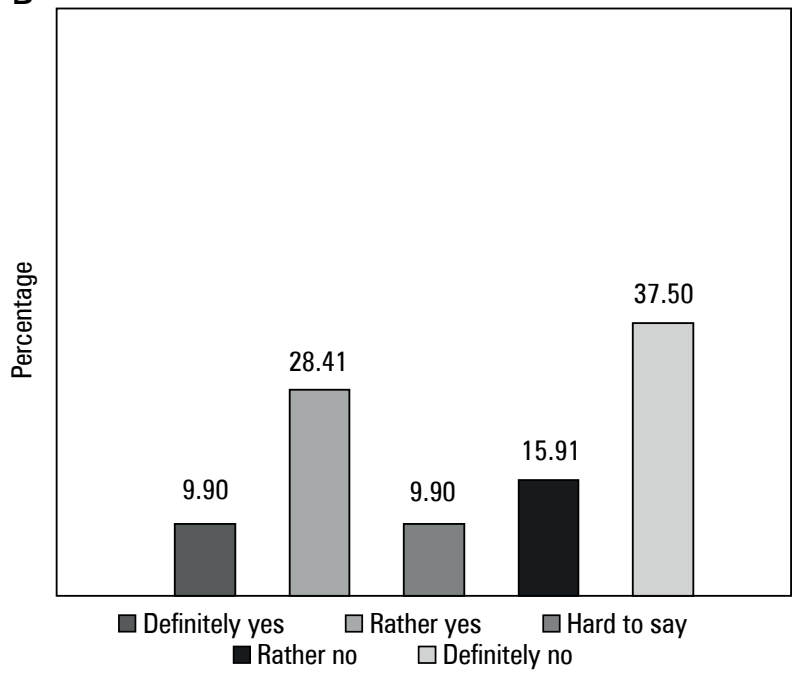

D

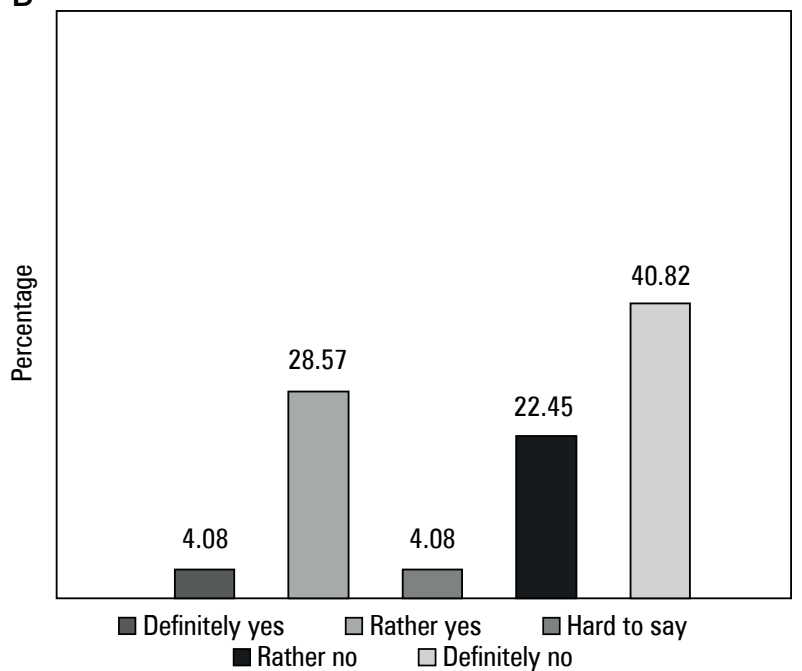

Figure 5. Attitude to the statement $I$ had sore nipples. Distribution of answers depending on the respondents' age $(n=257): \mathbf{A}-18-25$ years old, $\mathbf{B}$ - 26-30 years old, $\mathbf{C}$ - 31-35 years old, $\mathbf{D}->35$ years old

(24.1\%) followed by Rather yes (21.7\%). The total of $47.8 \%$ of the respondents said they had little breast milk, while $43.1 \%$ denied the statement. Statistically significant differences were observed depending on the respondents' age $(p 1=0.027769$, $p 1<a)$. The survey participants aged $18-25$ years most often denied having too little breast milk (37.93\%). Nevertheless, over $50 \%$ of them complained about not having enough breast milk (the total of answers Definitely yes and Rather yes). Women over 35 years of age most often denied having too little breast milk (34.07\% - the total of answers Definitely not and Rather not). Over $50 \%$ of women in this age group complained about having too little breast milk (Figure 4). Another statement that the respondents were asked to reply to was I had sore nipples. Definitely not was the most common answer (35.6\%). Definitely yes was another common answer (26.5\%). In total $39.5 \%$ of the respondents said they had sore nipples, while $53.4 \%$ denied the statement (the total of answers Definitely yes and Rather yes, and Definitely not and Rather not). However, no statistically significant differences were observed between socio-demographic factors and oc- currence of sore nipples (Table I). Nonetheless, nearly 60\% of the youngest women confirmed that they suffered from sore nipples (the total of answers Definitely yes and Rather yes). Women over 35 years of age most often denied suffering from sore nipples (40.82\%) (Figure 5). The respondents with primary and basic education most often complained about sore nipples (66.66\% - the total of answers Definitely yes and Rather yes) (Figure 6). The last question concerned difficulty in getting the baby to latch. The respondents were asked to express their attitudes to the statement It was difficult to get the baby to latch. $12.06 \%$ definitely confirmed having had such a problem, $16.73 \%$ gave the answer Rather yes, $31.53 \%$ definitely denied having had the problem, while $27.24 \%$ answered Rather no. No statistically significant differences between socio-demographic factors and occurrence of problems with getting the baby to latch were discovered (Table I). Nevertheless, it was observed that the respondents from the age group 26-30 were the ones who most often confirmed having problems with getting the baby to latch (54.84\%) (Figure 7). 
A

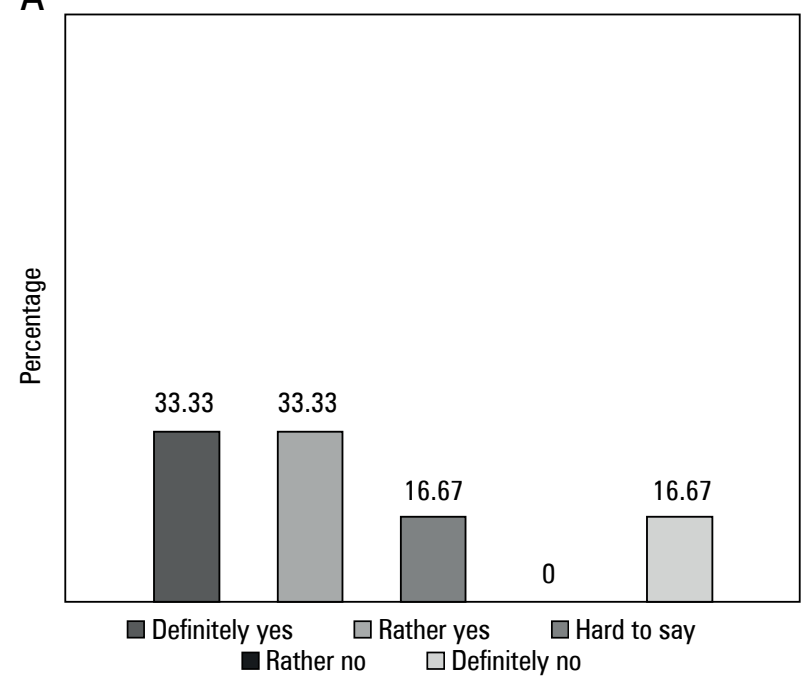

C

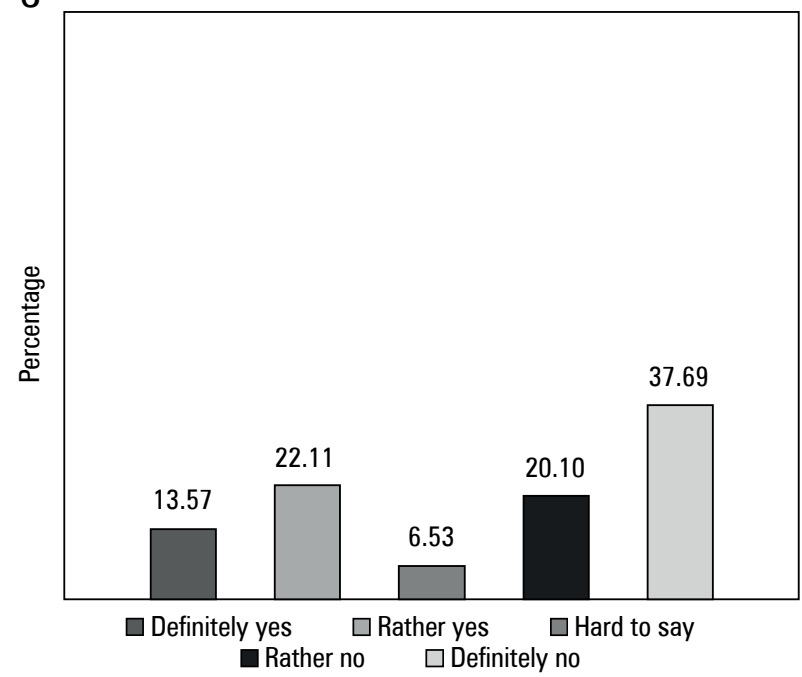

\section{Discussion}

Lactation problems are among the main reasons for stopping breastfeeding [10-13]. The range of problems is wide, while the most common ones among Polish women include sore nipples, breast milk scarcity, and difficulty in getting the baby to latch $[14,15]$. Women living in other countries report similar problems [16]. Thirty-five percent of women give breast milk scarcity as the main reason for stopping breastfeeding [9]. Nearly a half of the respondents reported the issue in the author's own study. The situation can definitely be called a public health problem. The scale of the phenomenon seems to be too wide. According to Gatti, the fact that mothers report breast milk scarcity so often can be caused by deficits in knowledge of the issue. Mothers treat their baby's satisfaction as the major indicator forgetting that a newborn's crying and anxiety may not necessarily be caused by hunger. A reliable assessment intended to confirm the actual breast milk scarcity is very often not performed [9]. The researcher points out that the topic of
B

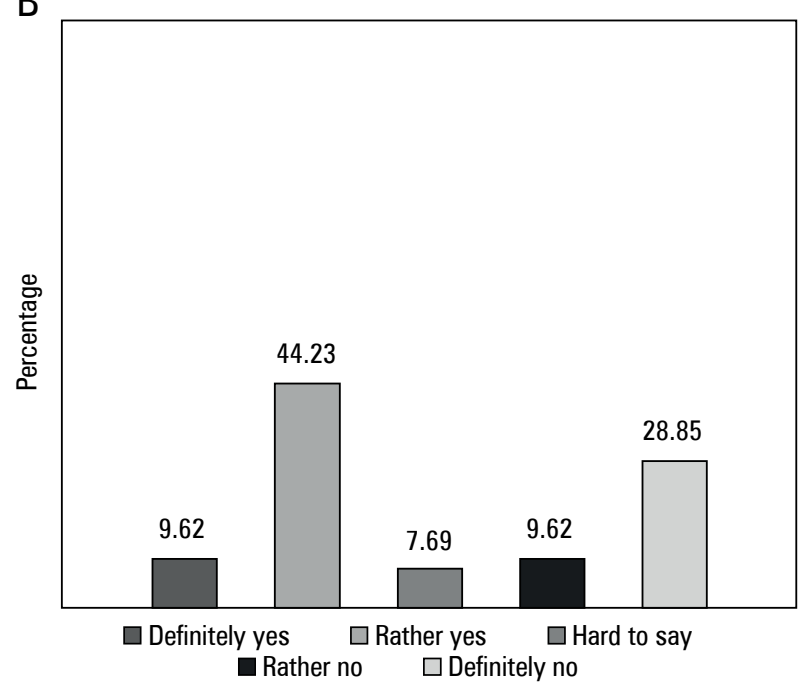

Figure 6. Attitude to the statement I had sore nipples. Distribution of answers depending on the respondents' education $(n=257)$ : A - primary/basic vocational, B - secondary, C - tertiary

the relationship between insufficient breast milk production and socio-demographic factors is not mentioned in the literature frequently enough [9]. Barbosa et al. share a similar opinion. They also highlight the finding that very young mothers belong to the group threatened with the risk of not providing sufficient breast milk to their babies [17]. The opinion contradicts the results of the studies in which the oldest respondents (over 35 years of age) were the ones who least often denied that they experienced breast milk scarcity. Similar findings were published by Nommsen-Rivers et al. In their opinion, the mother's age of over 30 years is a risk factor of delayed lactogenesis, which may result in an impression of insufficient milk production [18]. In relation to the fact that a huge number of respondents reported breast milk scarcity, the issue emerges of the percentage of mothers who supplementarily feed their babies with modified milk. The study revealed that as many as $70 \%$ of the respondents confirmed such practice, which is a worrying result in light of the findings that supplementary feeding of newborns contributes to the decision to stop breastfeeding prematurely 
A

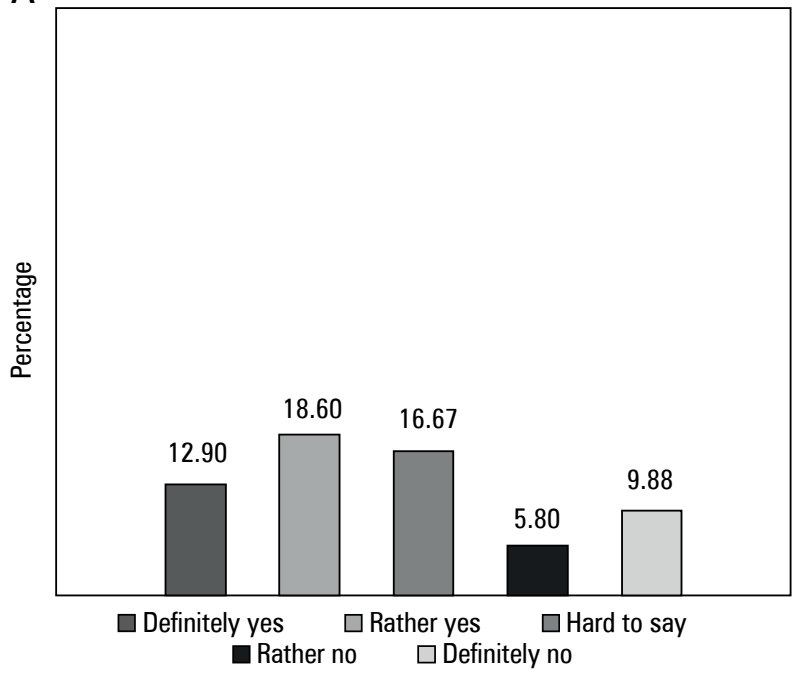

C

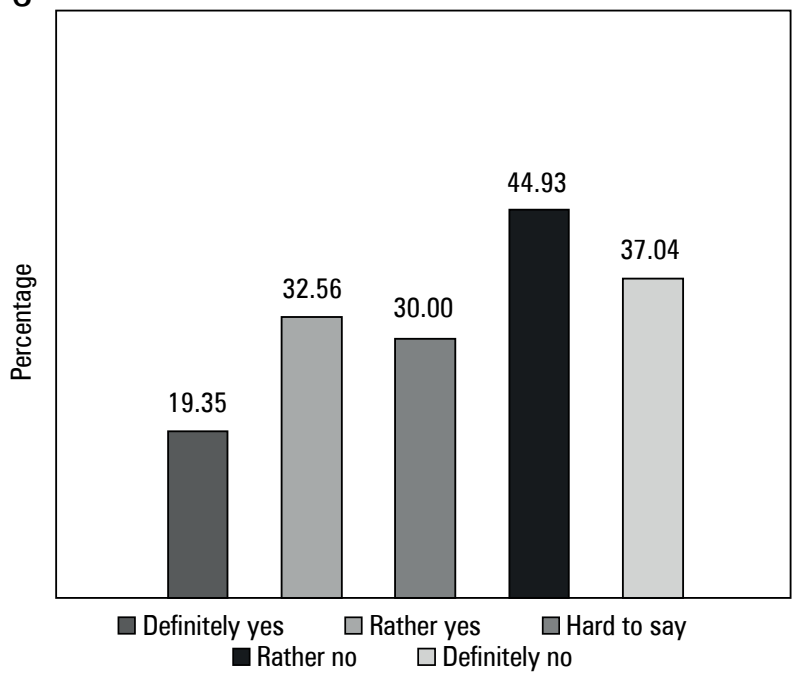

B



D

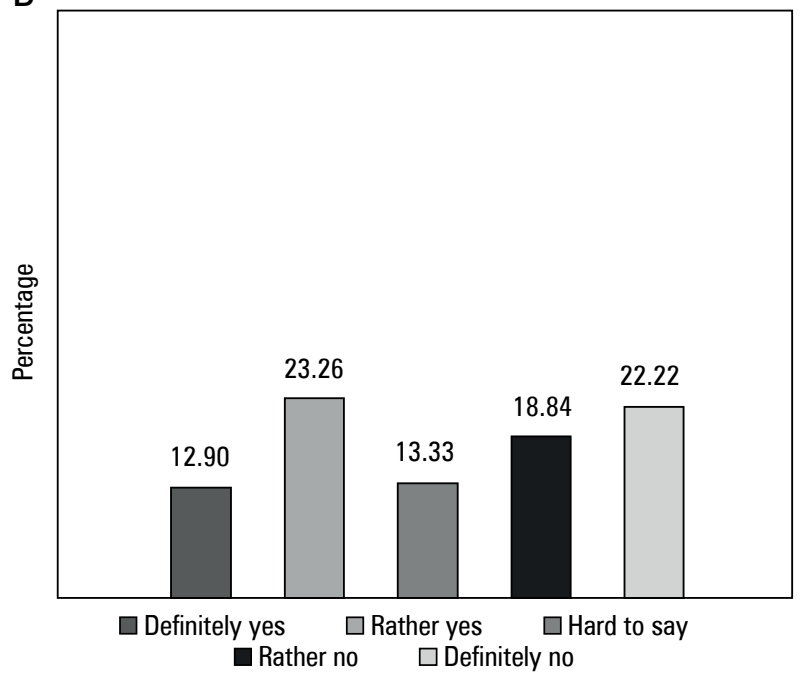

Figure 7. Attitude to the statement It was difficult to get the baby to latch. Distribution of answers depending on the respondents' age $(n=257)$ : A - 18-25 years old, $\mathbf{B}-26-30$ years old, $\mathbf{C}-31-35$ years old, $\mathbf{D}->35$ years old

$[19,20]$. Women living in a town/city with a population over 50,000 people supplementarily fed their babies significantly more often. Similar results were obtained by Qiu et al., who carried out studies in China. The indices of breastfeeding only upon leaving the hospital were definitely higher among women living in suburban and rural areas [21]. Scientists from south-eastern Nigeria observed that the indicators of breastfeeding only were lower among women with a higher socio-economic status. In the scientists' opinion, it can be attributed to the fact that the possibility of feeding a baby with modified milk is perceived as a symbol of social status because it entails higher financial expenditure [22]. Despite the fact that the studies apply to a developing country, which represents a culture different from that in Poland, one can risk saying that the higher percentage of supplementarily fed babies among the inhabitants of larger towns/cities is related to their higher socio-economic status. Further studies are required in the field.

There are common literature findings which claim that longer breastfeeding is related to the mother's more mature age, higher level of education, and higher socio-economic status $[6,8,16,23]$. More frequent difficulty in the early stages of lactation is attributed to younger mothers [17]. The results of own studies contradict the aforementioned findings. Women aged 31-35 years were the ones to report lactation problems most often. In the case of respondents in the age group 18-35 years the frequency of occurrence of the problems was directly proportional to the mothers' age. The respondents in the youngest age group (18-25 years) confirmed experiencing lactation problems least often. It is worth paying attention to the fact that the Clinical Protocol of the Academy of Breastfeeding Medicine mentioned both very young and advanced mother's age as risk factors of lactation problem occurrence [24]. Interestingly enough, the respondents in the youngest age group most often experienced sore nipples. The respondents over 35 years of age represented the group that most often denied suffering from the ailment. Turkish researchers reached similar conclusions - in their opinion, age below 25 years is a risk factor for nipple harm and soreness. In the group they studied women below 35 years old reported having the problem much more often than other respondents [25]. In our 
own study, women with vocational or basic education most often complained about sore nipples. At the same time, respondents in the group reported lactation problems far less often than those with secondary or tertiary education. The answers given by respondents with the lowest level of education were only extreme (Definitely yes/no, with no answers Rather yes $/$ no). As many as $50 \%$ of the women were not able to give the answer to the question concerning occurrence of breastfeeding problems (Hard to say answers). This may be related to the fact that due to a low knowledge level of breastfeeding the women were not able to identify the situations that are named as lactation problems. A study by Zielińska et al. revealed that women with vocational education have more limited knowledge of lactation [26]. Amir and Donath pointed out that women from families with lower income have limited possibility to seek help in case of lactation difficulty [8]. Perhaps it is also the key to explaining why older and better educated women report breastfeeding difficulty more often. Older women with a higher level of education can have greater knowledge, and that is why they may find it easier to identify the situation as a problem.

Moreover, the women are able to respond quicker and more effectively in case of failures and count on support from the people in their environment. That is the most probable reason why the majority of researchers confirm that women representing the groups breastfeed their babies longer.

\section{Conclusions}

Socio-demographic conditions are related to the behaviour that affects breastfeeding. Such factors as the mother's age and education level seem to be of paramount importance. Both young and mature women need support and education because the groups experience different problems caused by various factors. Mothers with the lowest education level should be provided with special care.

It is necessary to carry out further studies in the area considering groups of mothers more diversified for their education and place of residence.

\section{Conflict of interest}

The authors declare no conflict of interest.

\section{References}

1. American Academy of Pediatrics. Section on breastfeeding. Breastfeeding and the use of human milk. Pediatrics 2012. Access from: http://pediatrics.aappublications.org/content/early/2012/02/22/ peds.2011-3552.full.pdf+html

2. Szajewska H, Socha P, Horvath A, et al.; Polish Society for Paediatric Gastroenterology, Hepatology and Nutrition. Nutrition of healthy term infants. Recommendations of the Polish Society for Paediatric Gastroenterology, Hepatology and Nutrition. Stand Med Pediatria 2014; 11: 321-38.

3. CnoL (Centre for Knowledge of Lactation), Breastfeeding in Poland, 2015 Report, [quoted: 19 February 2017, 16:50], Access from: http://femaltiker.pl/wp-content/uploads/2015/05/Raport_ Karmienie_Piersia_w_Polsce_20151.pdf
4. Butler S, Williams M, Tukuitonga C, Paterson J. Factors associated with not breastfeeding exclusively among mothers of a cohort of Pacific infants in New Zealand. N Z Med J 2004; 177: 1-11.

5. Prior E, Santhakumaran S, Gale C, Philipps LH, Modi N, Hyde MJ. Breastfeeding after caesarean delivery: a systematic review and meta-analysis of world literature. Am J Clin Nutr 2012; 95: 1113-5.

6. Meedya S, Fahy K, Kable A. Factors that positively influence breastfeeding duration to 6 months: a literature review. Women Birth 2010; 23: 135-45.

7. van Rossem L, Oenema A, Steegers EA. Are starting and continuing breastfeeding related to educational background? The generation R study. Pediatrics 2009; 123: 1017-27.

8. Amir LH, Donath SM. Socioeconomic status and rates of breastfeeding in Australia: evidence from three recent national health surveys. Med J Aust 2008; 189: 254-6.

9. Gatti L. Maternal perceptions of insufficient milk supply in breastfeeding. J Nurs Scholarsh 2008; 40: 355-63.

10. Ahluwalia IB, Morrow B, Hsia J. Why do women stop breastfeeding? Findings from the pregnancy risk assessment and monitoring system. Pediatrics 2005; 116: 1408-12.

11. Li R, Fein SB, Chen J, Grummer-Strawn LM. Why mothers stop breastfeeding: mothers' self-reported reasons for stopping during the first year. Pediatrics 2008; 122: 69-76.

12. Odom EC, Li R, Scanlon KS, Perrine CG, Grummer-Strawn L. Reasons for earlier than desired cessation of breastfeeding. Pediatrics 2013; 131: 726-32.

13. de Jager M, Hartley K, Terrazas J, Merrill J. Barriers to breastfeeding - a global survey on why women start and stop breastfeeding. Eur J Obstet Gynecol 2012; 7 (Suppl. 1): 25-30.

14. Józefów P, Przestrzelska M, Knihinicka-Mercik Z. The course of lactation in the early puerperium after natural birth and by caesarean section including parity of woman. Piel Zdr Publ 2013; 3: 133-42.

15. Bączek G, Golubińska H, Dmoch-Gajzlerska E. Selected problems of delivery period-the role of family midwife's. Med Rev 2012; 2 : 200-12.

16. Schwartz K, D’Arcy HJ, Gillespie B, Bobo J, Longeway M, Foxman B. Factors associated with weaning in the first 3 months postpartum. J Fam Pract 2002; 51: 439-44.

17. Barbosa GEF, da Silva VB, Pereira JM, et al. Initial breastfeeding difficulties and association with breast disorders among postpartum women. Rev Paul Pediatr 2017; 35: 265-72.

18. Nommsen-Rivers LA, Chantry CJ, Peerson JM, Cohen RJ, Dewey KG. Delayed onset of lactogenesis among first-time mothers is related to maternal obesity and factors associated with ineffective breastfeeding. Am J Clin Nutr 2010; 92: 574-84.

19. Chantry CJ, Dewey KG, Peerson JM, Wagner EA, Nommsen-Rivers LA. In-hospital formula use increases early breastfeeding cessation among first-time mothers intending to exclusively breastfeed. J Pediatr 2014; 164: 1339-45.

20. Isaia M, Theodorou M, Galanis P, Nikolentzos A, Polyzos N. Breastfeeding determinants in Cyprus: a cross-sectional study. J Neonatal Nurs 2017; 23: 267-74.

21. Qiu L, Zhao Y, Binns CW, Lee AH, Xie X. A cohort study of infant feeding practices in city, suburban and rural areas in Zhejiang Province, PR China. Int Breastfeed J 2008; 3: 4.

22. Onah S, Osuorah DIC, Ebenebe J, Ezechukwu C, Ekwochi U, Ndukwu I. Infant feeding practices and maternal socio-demo- 
graphic factors that influence practice of exclusive breastfeeding among mothers in Nnewi South-East Nigeria: a cross-sectional and analytical study. Int Breastfeed J 2014; 9: 6.

23. Whipps MD. Education attainment and parity explain the relationship between maternal age and breastfeeding duration in U.S. mothers. J Hum Lact 2017; 33: 220-4.

24. Evans A, Marinelli KA, Taylor JS. ABM Clinical Protocol \#2: Guidelines for Hospital Discharge of the Breastfeeding Term Newborn and Mother: "The Going Home Protocol," Revised 2014. Breastfeed Med 2014; 9: 3-8.

25. Şahin H, Yllmaz M, Aykut M, Balcı E, Sağıroğlu M, Öztürk A. Risk factors for breastfeeding problems in mothers who presented to two public healthcare centers in Kayseri province. Turk Arch Ped 2013; 48: 145-51.

26. Zielińska MA, Sobczak A, Hamułka J. Breastfeeding knowledge and exclusive breastfeeding of infants in first six months of life. Rocz Panstw Zakl Hig 2017; 68: 51-9. 